\title{
Application of statistical techniques and artificial neural network to estimate force from sEMG signals
}

\author{
V. Khoshdel and A. Akbarzadeh* \\ Center of Excellence on Soft Computing \& Intelligent Information Processing, Mechanical Engineering Department, Ferdowsi University of \\ Mashhad, Mashhad. \\ Received 20 April 2015; Accepted 21 February 2016 \\ *Corresponding author: Alireza.Akbarzadeh@um.ac.ir(A. Akbarzadeh).
}

\begin{abstract}
This paper presents an application of the design of experiment(DoE) techniques to determine the optimized parameters of the artificial neural network (ANN)model, which are used to estimate the force from the electromyogram (sEMG) signals. The accuracy of the ANN model is highly dependent on the network parameter settings. There are plenty of algorithms that are used to obtain the optimal ANN settings. However, to the best of our knowledge, no regression analysis has yet been used to model the effect of each parameter as well as presenting the percent contribution and significance level of the ANN parameters for force estimation. In this paper, the sEMG experimental data is collected, and the ANN parameters are regulated based on an orthogonal array design table to train the ANN model. The Taguchi method helps us to find the optimal parameters settings. The analysis of variance (ANOVA) technique is then used to obtain the significance level as well as the contribution percentage of each parameter I order to optimize ANN' modeling in the human force estimation. The results obtained indicate that DoE is a promising solution to estimate the human force from the sEMG signals.
\end{abstract}

Keywords: Artificial Neural Network, Taguchi Method, Analysis of variance, EMG signals.

\section{Introduction}

The rehabilitation robot has recently received much attention by the physiotherapists and robot researchers. There are some reasons for this increasing attention. The rehabilitation robot can consistently apply therapy over long time periods without tiring. In addition, the use of sensors can highly improve the quality of therapy. Moreover, it can provide some types of therapy exercises that a therapist cannot do. Furthermore, the robot can decrease the cost of a physiotherapy process. There is also a great need from many patients with movement disabilities to have session physiotherapy. Finally, the rehabilitation robot can be easily programmed by a physiotherapist to perform the suggested exercises [1]. Muscle activity can be recorded from selected muscles using surface EMG electrodes (sEMG), while the user moves his arm. The muscular activity can be transformed to the force and kinematic variables that are used as the inputs in the robot control by a decoding procedure.

The sEMG signals are one of the most common biological signals thaht help us in the robot control according to the user's intention. The
sEMG signals can directly reflect the muscle activation level in real time [2-8]. In voluntary movements, force is associated with the motor unit recruitment and variations in the motor unit firing frequency [9]. At the same muscle length and under the isometric conditions, a greater number of recruited motor units with greater discharge frequencies (i.e. muscle activation) lead to a greater force generation. Therefore, a linear relationship between EMG and muscle force is assumed. Although, Precise estimation of muscle force based on the sEMG signals in real time provides valuable information for a robot control system in order to perform effective therapeutic exercises, while the sEMG signals are random, continuous, and non-linear in nature [10, 11]. Therefore, the sEMG signals should be processed in order to get a simple model for its amplitude and then map this amplitude for joint force. Various methods have been proposed for the sEMG-based force estimation such as mathematical models, artificial neural network (ANN), and neurofuzzy. It has been shown that ANN will yield efficient to estimate the voluntary 
limb force [12]. It has been shown that the setting of network parameters, such as the number of neurons, number of hidden layers, and learning rate have great influences on the accuracy of ANN model. However, selection of the ANN design parameters is still an open question in the force estimation for the rehabilitation robots. Design of experiment (DoE), as a statistical technique, is widely used to study the relationship between the factors involved, and affecting the outputs of the process. Additionally, it can be used to systematically identify the optimum setting of factors to reach the desired output. In this paper, we used DoE to find the best setting of the ANN parameters in order to achieve a minimum error in force estimation. The applications of DoE techniques to optimize the ANN parameters have been reported in the literature [13-18]. It has been found that some factors such as the number of neurons in the hidden layers, transfer function, and training function have significant effects on the ANN performance. Therefore, in this paper, the sEMG experimental data was collected, and the ANN parameters were regulated based on an orthogonal array design table to train the ANN model. The Taguchi method helps us to find the optimal parameter settings. The analysis of the variance (ANOVA) technique is then used to obtain the significance level as well as the contribution percentage of each parameter to optimize the ANN modeling in the human force estimation. The results of human force estimation have indicated that DoE is a promising solution to optimize the ANN modeling. This paper is organized as followss: Section 2 explain how ANN is used for the sEMG-based force estimation. The Taguchi method is introduced in section 3. In section 4, the experimentation procedure is presented. The results obtained and the concluding discussion are presented in sections 5 and 6 .

\section{Force estimation}

In the control system of the rehabilitation robots, the estimated force is used as an input signal. Nevertheless, the raw sEMG signals are not suitable as input signals for controllers, and must be processed prior to use. In this paper, each EMG channel is independently processed in three steps, as follows. Step 1: The raw EMG signals must be filtered. In this step, a a $5^{\text {th }}$ ordered notch filter is used to remove the $60 \mathrm{~Hz}$ noise resulting from the power supply. Step 2: The EMG signal should be rectified. The absolute value for the EMG signals is calculated in this step. Step 3: The online moving average (OMA) of the rectified EMG signals is calculated as follows:

$$
E(t)=\sqrt{\frac{1}{N} \sum_{i=0}^{N} E(t-i)^{2}}
$$

where, $N$ is the number of segments $(N=100)$ and $E(t)$ is the value of the rectified EMG at its sampling point. The signal processing procedure is shown in figure 1. Finally, the processed EMG (PEMG) signals are ready to be used as an input for the ANN estimator.

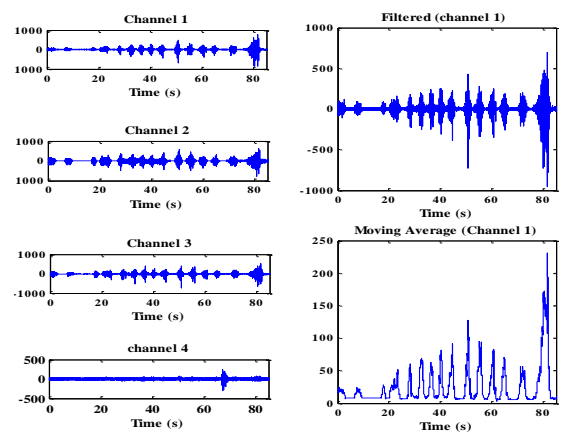

Figure 1. EMG process for neural network estimation.

MLP and cascade are the two most common ANN structure that are used for force estimation. The detailed structural design for te feed-forward condition for MLP and Cascade is depicted in figure 2 .

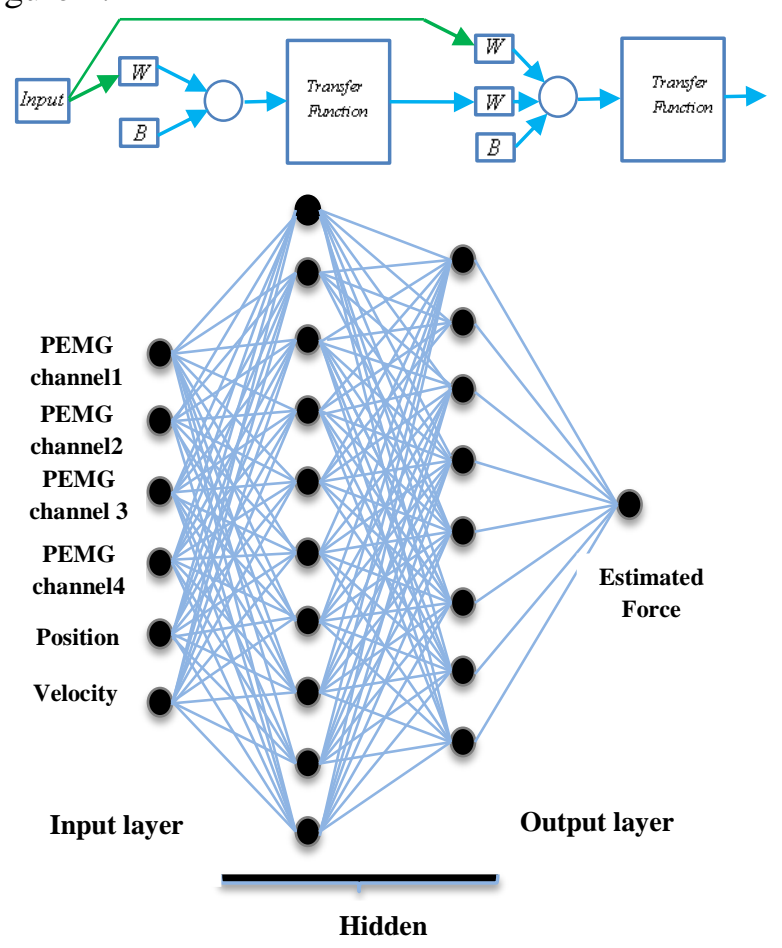

Figure 2. Cascade (up) and MLP (down) neural network structure. 
We proposed the PEMG signals and the of human dynamics information as the inputs for estimation. Hence, the relationship between the sEMG signals and the human force can been proposed as:

$$
\mathbf{F}_{\mathrm{h}_{\mathrm{est}}}=\left[\begin{array}{c}
P E_{c h 1}(t) \\
P E_{c h 2}(t) \\
P E_{c h 3}(t) \\
P E_{c h 4}(t) \\
\theta \\
\dot{\theta}
\end{array}\right] W_{6 \times n}^{h l 1} W_{n \times m}^{h l 2} W_{m \times 1}^{o l}
$$

where, $F_{h_{e s t}}$ is the estimation of human force, $P E_{c h i}(t)$ is the processed EMG signal for the $\mathrm{i}^{\text {th }}$ channel, and $\theta$ and $\dot{\theta}$ are the angular position and velocity, respectively. $W_{6 \times n}^{h l 1}$ and $W_{6 \times n}^{h l 2}$ are the neural network weight matrices of the hidden layers, and $W_{6 \times n}^{o l}$ is the weight matrix of the output layer.

Selection of the ANN design parameters is still an open challenge. For example, the network performance may deteriorate by a large number of hidden neurons. In addition, to store the huge numbers of network variables, a huge memory is required, and hence, training becomes complicated. However, the network cannot adjust the weight and bias properly during training, if a very low number of neurons are selected in the hidden layer, which result in over-fitting. Overfitting makes the network excessively complex, and thus, the non-generalized network generates random error, and provides a very poor classification. Figure3 depicts the design parameters of ANN that largely affect the ANN performance.

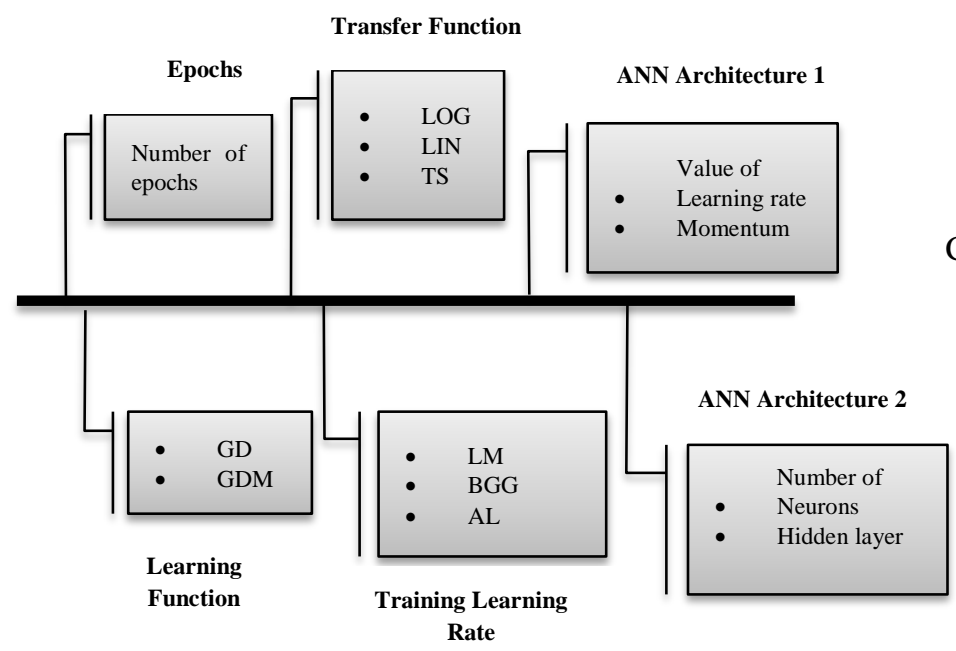

Figure 3. Design parameters of ANN.

Due to the lack of a specific rule for finding the optimal ANN parameters for an optimized network performance, a statistical technique is performed to investigate the appropriate network topology for force estimation.

\section{Taguchi Method}

In the Taguchi method, constructed on the fractional factorial experiment, the independent parameters are divided into design parameters and noise parameters [19]. The design parameters are those for which the designer chooses values as a part of the design process and the noise parameters are defined for modelling uncertainty in the design.

In the design process, we choose the design parameter values such that the design goals are maximized despite the noise parameter actions. This means that a robust tuning technique using the Taguchi method would enable the regulators not only to reduce the control errors but also to decrease variations in those values while remaining insensitive to changes in system dynamics and variations in operating points.

By implementing this approach, the researchers can significantly decrease the time required for experimental investigations. DoE using the Taguchi method is briefly outlined below [20]:

A. Identification of objectives: the first step of the Taguchi method is to identify a specific objective. In this paper, the objective is the ANN parameters, which are used to minimize the estimation error.

B. Determining the quality characteristic: in Taguchi method, the quality characteristics are classified into one of the following three types: nominal-the-best, smaller-the-better, and larger-the-better. In this study, the smaller-thebetter type is used to decrease the estimation error.

C. Selecting the controllable factors and noise factors: one of the most important steps involved is the selection of the factors to be tested for their influence on the quality characteristic. Actually, if we select the controllable factors and noise factors carelessly, this can lead to false conclusions and may require the experiments to be repeated. The desired number of levels must be determined after selecting the factors. In this paper, the ANN parameters are used as the controllable factors, and we do not have any noise factor. The numbers of levels for the ANN parameters are defined in table 2. In the 
next step, we should assign a physical value to each level of controllable factors.

D. Selecting an orthogonal array: it is clear that testing all combinations of the factor levels is required in the full factorial experiment. For example, a study involving 6 factors with three levels for each factor would require $3^{\wedge} 4 \times 2^{\wedge} 2=324$ experiments. Orthogonal arrays produce smaller and less-costly experiments. Using an L_27 ( $\left.3^{\wedge} 5\right)$ orthogonal array, for example, a study involving 5 factors with three levels for each factor can be conducted with only 27 experiments. In addition to being efficient, the procedures used for using orthogonal arrays are straightforward and easy to use. In this paper, in order to reduce the number of experiments, an L_36 $\left(3^{\wedge} 6\right)$ orthogonal array is selected. This array implies only 36 experiments.

E. Conducting the experiment and analysis: conducting the experiments includes execution of the experiment as developed in the planning and design phases. The analysis phase of experimentation is related to calculations for converting raw data into the representative signal-to-noise ratio (SNR). SNR, as a measurement tool for determining robustness, is a critical component to an optimal parameter design. SNR can be adopted as the index of the system ability to perform well regardless of the noise by including the impact of noise factors on the process. By successfully applying this concept to experimentation, it is possible to determine the design parameters of ANN that can produce the minimum estimation errors while maximizing SNR.

In the case of the smaller-the-better quality characteristic, SNR can be written as Eq. 3:

$$
I T A E=\int F_{m}-F_{e s t} d t
$$

where, $F_{m}$ is the measured force, $F_{\text {est }}$ is the estimated force, and ITEA is the integral force error. Consequently, SNR can be defined as:

$\mathrm{SNR}=-10 \log I T A E$

The Taguchi method reduces the number of experiments over the full-factorial approach. Therefore, to provide the levels of confidence in the results, it is valuable to use the statistical analysis of experiments, which are called the analysis of variance (ANOVA). Furthermore, ANOVA identifies and ranks the variables that affect the variance of the output signal. ANOVA is one of the main steps in using the Taguchi method.

\section{Experimental \\ 4.1 Setup and SEMG data collection}

Four channels of sEMG signals are used as the main input signals in order to estimate the participants real force. Locations of the sEMG electrodes are shown in figure 4 . Each channel mainly corresponds to one muscle, as shown in table 1 . To determine the magnitude of the sEMG signals of the knee extensors, our participants were seated on a dynamometer (Biodex System3, Biodex Medical Systems Inc., USA) with a hip angle of $85^{\circ}$.
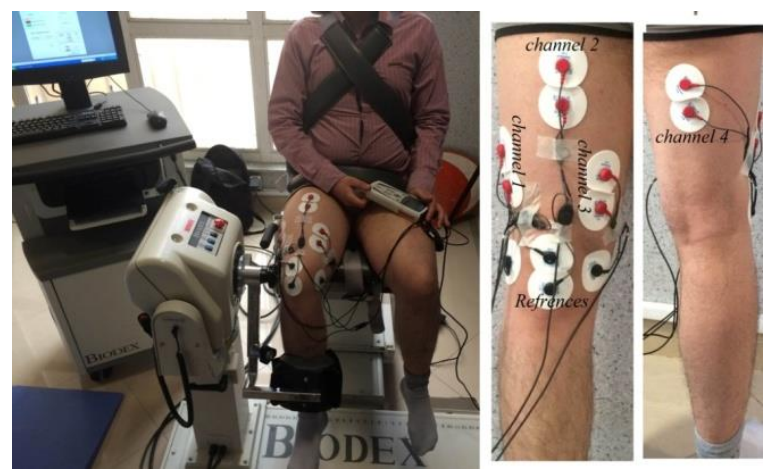

Figure 4. The locations of sEMG electrodes.

Table 1 . Muscles for each sEMG channel.

\begin{tabular}{cllll}
\hline $\begin{array}{c}\text { EMG } \\
\text { Channel }\end{array}$ & Ch-1 & Ch-2 & Ch-3 & Ch-4 \\
\hline \multirow{2}{*}{ Muscle } & & & & \\
& Vastus & Rectus & Vastus & Bicep \\
& Lateralis & Femoris & Medialist & Femoris \\
\hline
\end{tabular}

Electrodes $(\mathrm{Ag} / \mathrm{AgCl})$ with an electrolytic gel interface were positioned above the midpoint of the muscle belly (with $2 \mathrm{~cm}$ distance on interelectrodes) of the Rectus Femoris, Vastus Lateralis, Vastus medialis, and Biceps Femoris (Figure 3). Moreover, the reference electrodes were located on the patella bone. The skin was carefully shaved and cleaned with alcohol in order to reduce the skin impedance. To reduce the motion artefacts of the electrodes, they were further secured to the skin with an elastic tape, together with the preamplifier. Prior to the experiment, the leg was passively shaken to check the mechanical artefacts of the sEMG signals from each muscle. Several tests (e.g. contractions against manual resistance in knee flexion and extension) were performed to visualize whether a good signal was produced from each muscle. When artefacts or poor signals were observed, the preparation procedure was repeated. ME6000 was used to record the sEMG signal from muscle in 
Sport Sciences Research Institute of IRAN (SSRI). The data collected from the EMG sensor was sampled using a $2000 \mathrm{~Hz}$ sampling frequency.

\subsection{Design of experiments(DoEs)}

The experimental region was decided as per Taguchi design approach. The number of levels for each controllable process parameter was provided in table 2. A wide experimental region was covered so that the sensitivity to noise factors did not change with small variations in these factor settings, and to obtain optimum regions for the process parameters. Therefore, each parameter was analysed at different levels of the ANN parameters. The cascade-forward backpropagation and MLP back-propagation ANN with three training functions(LM, BoydonFletcher - Goldfarb - Shanno, GDA) were considered in this study. The number of neurons was varied from 1 to 10 and the number of hidden layer was varied from 2 to 4 . Three transfer functions (PURELIN, LOGSIG, and TANSIG) were used in this work. The two different learning functions gradient descent (GD) and gradient descent with momentum (GDM), frequently used for ANN were also considered. These factors and their levels used for DoE are shown in table 2. After deciding the parameters and their levels, the Taguchi design approach was applied to decide the experimental region. An orthogonal array L_36, which was designed for these factors is shown in table 3.

Table 2. ANN parameters levels.

\begin{tabular}{|c|c|c|c|c|}
\hline & & \multicolumn{3}{|c|}{ Levels } \\
\hline Parameters & Range & 1 & 2 & 3 \\
\hline Learning Function(LF) & - & GD & GDM & - \\
\hline Training Function $(\operatorname{TrF})$ & - & LM & BFGS & GDA \\
\hline $\begin{array}{l}\text { Number of hidden } \\
\text { Layers(NL) }\end{array}$ & $2-4$ & 2 & 3 & 4 \\
\hline $\begin{array}{l}\text { Number of } \\
\text { neurons }(\mathrm{Nn})\end{array}$ & $1-10$ & 1 & 5 & 10 \\
\hline Topology of ANN(TP) & - & MLP & Cascade & \\
\hline Transfer Function(TF) & - & TAN & PUR & LOG \\
\hline
\end{tabular}

The experiments were conducted as per L_36 orthogonal array for the set of parameters shown in table 3. MSE was calculated for all trials as per the L_36 orthogonal array to understand the process parameter characteristics and optimum setting of user-defined parameters.

By plotting the average response value for each factor level (Figure 5), relative comparisons of the slope between the points plotted can be made.
Table 3. Orthogonal for different factors of ANN.

\begin{tabular}{|c|c|c|c|c|c|c|c|}
\hline $\mathrm{NO}$ & TP & LF & $\mathrm{TrF}$ & NL & $\mathrm{Nn}$ & $\mathrm{TF}$ & ITAE \\
\hline 1 & 1 & 1 & 1 & 1 & 1 & 1 & 215.4 \\
\hline 2 & 1 & 1 & 2 & 2 & 2 & 2 & 220.0 \\
\hline 3 & 1 & 1 & 3 & 3 & 3 & 3 & 251.5 \\
\hline 4 & 1 & 1 & 1 & 1 & 1 & 1 & 215.4 \\
\hline 5 & 1 & 1 & 2 & 2 & 2 & 2 & 220.0 \\
\hline 6 & 1 & 1 & 3 & 3 & 3 & 3 & 251.5 \\
\hline 7 & 1 & 1 & 1 & 1 & 2 & 3 & 236.6 \\
\hline 8 & 1 & 1 & 2 & 2 & 3 & 1 & 183.4 \\
\hline 9 & 1 & 1 & 3 & 3 & 1 & 2 & 223.7 \\
\hline 10 & 1 & 2 & 1 & 1 & 3 & 2 & 252.3 \\
\hline 11 & 1 & 2 & 2 & 2 & 1 & 3 & 216.4 \\
\hline 12 & 1 & 2 & 3 & 3 & 2 & 1 & 246.7 \\
\hline 13 & 1 & 2 & 1 & 2 & 3 & 1 & 551.0 \\
\hline 14 & 1 & 2 & 2 & 3 & 1 & 2 & 205.9 \\
\hline 15 & 1 & 2 & 3 & 1 & 2 & 3 & 215.1 \\
\hline 16 & 1 & 2 & 1 & 2 & 3 & 2 & 216.3 \\
\hline 17 & 1 & 2 & 2 & 3 & 1 & 3 & 215.0 \\
\hline 18 & 1 & 2 & 3 & 1 & 2 & 1 & 213.8 \\
\hline 19 & 2 & 1 & 1 & 2 & 1 & 3 & 176.2 \\
\hline 20 & 2 & 1 & 2 & 3 & 2 & 1 & 170.6 \\
\hline 21 & 2 & 1 & 3 & 1 & 3 & 2 & 249.5 \\
\hline 22 & 2 & 1 & 1 & 2 & 2 & 3 & 183.8 \\
\hline 23 & 2 & 1 & 2 & 3 & 3 & 1 & 694.5 \\
\hline 24 & 2 & 1 & 3 & 1 & 1 & 2 & 254.0 \\
\hline 25 & 2 & 1 & 1 & 3 & 2 & 1 & 321.2 \\
\hline 26 & 2 & 1 & 2 & 1 & 3 & 2 & 250.2 \\
\hline 27 & 2 & 1 & 3 & 2 & 1 & 3 & 211.3 \\
\hline 28 & 2 & 2 & 1 & 3 & 2 & 2 & 317.9 \\
\hline 29 & 2 & 2 & 2 & 1 & 3 & 3 & 245.3 \\
\hline 30 & 2 & 2 & 3 & 2 & 1 & 1 & 187.7 \\
\hline 31 & 2 & 2 & 1 & 3 & 3 & 3 & 213.1 \\
\hline 32 & 2 & 2 & 2 & 1 & 1 & 1 & 188.9 \\
\hline 33 & 2 & 2 & 3 & 2 & 2 & 2 & 199.2 \\
\hline 34 & 2 & 2 & 1 & 3 & 1 & 2 & 555.2 \\
\hline 35 & 2 & 2 & 2 & 1 & 2 & 3 & 247.6 \\
\hline 36 & 2 & 2 & 3 & 2 & 3 & 1 & 294.6 \\
\hline
\end{tabular}

With this best combination of design parameters, SNR increased to about $100 \%$. The results obtained were confirmed by the ANOVA analysis.

The percentage contributions are shown in table 4 . The main purpose of this analysis was to estimate the effect of each factor on the results. 


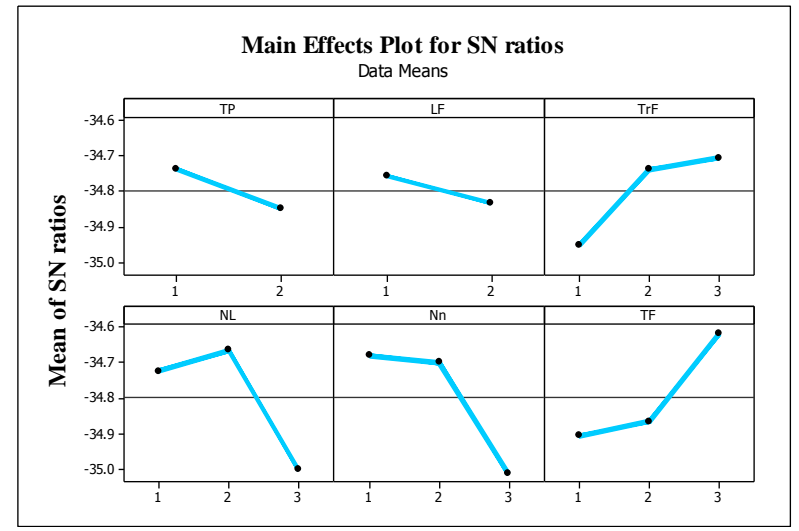

Figure 5. Main effects plot for SNRs.

Table 4. Percentage contribution.

\begin{tabular}{l|l|l}
\hline $\begin{array}{l}\text { Controller } \\
\text { Parameter }\end{array}$ & $\begin{array}{c}\text { Percentage } \\
\text { contribution }\end{array}$ & P-value \\
\hline TP & $12.01 \%$ & 0.236 \\
LF & $9.02 \%$ & 0.617 \\
Trf & $10.12 \%$ & 0.817 \\
NL & $19.42 \%$ & 0.138 \\
Nn & $19.01 \%$ & 0.147 \\
TF & $14.22 \%$ & 0.390 \\
ANOVA & $16.20 \%$ & \\
Error & & \\
\hline
\end{tabular}

The ANOVA analysis shows that $N L$ and $N n$ have the biggest percentage contributions. This confirms the results show in figure 5, which illustrates that SNR values for the levels of $N L$ and $N n$ differ significantly. It is important to note that the error contribution computed with ANOVA gives an idea of the confidence in the results.

P-values, which report the significance level of the parameters are shown in the second column of table 4 . The trend confirms that at stage 4 , while $\mathrm{NL}$ and Nn have minimum P-value, which means that these parameters significantly contribute toward ANN estimation, LF and $\mathrm{TrF}$ do not have important effects on the improvement of SNR.

To compare the proposed optimized ANN with manual tuning ANN parameters, the result of Simulink in MATLAB were compared. The Total error of estimation across 84 seconds was 3.45 for MATLAB manual tuning ANN and 6.32 for the proposed optimized ANN.

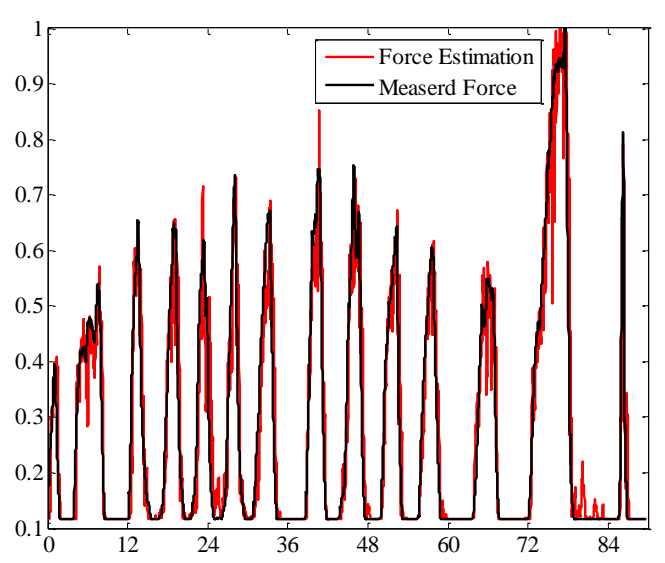

Figure 6. Force estimation by the optimized ANN.

We can vividly assert that the proposed method is more efficient than manual tuning.

The force estimated by the optimized ANN based on the Taguchi method is shown in figure 6, where the black line represents the measured force by load cells and red line represents the force estimated by ANN. As we can vividly realize, the performance of optimized ANN is well, and it can estimate the human force appropriately.

\section{Results and Discussion}

The highlights of this paper can be summarized as follows:

- Use of the DoE technique, presented in this paper, allow the researchers to obtain the optimum combinations of the ANN parameter settings with a minimal number of experiments among a large possible number of experiments. Using the Taguchi L_36 design, only 36 experiments were gathered and used to train the ANN model. The minimum number of experiments results in time and cost savings.

- By a close observation of the experimental table, it can be observed that the optimal combination settings suggested by the Taguchi method, is not any of the existing runs of the table.

- Verification runs, using the optimum ANN parameters, showed that the ANN model produced acceptable results.

- The authors believe that the optimum settings of the ANN parameters are largely problemdependent. Optimization process should be performed for each application, as the significant factors might be different for ANN trained for different purposes. 


\section{Conclusion}

The objective of this paper was to use the Taguchi method to determine the optimal ANN parameters for the human force estimation. To the best of the author's knowledge, application of the statistical technique and ANN to estimate human force from the sEMG signals as not yet been reported in the literature. Using an L_36 orthogonal array, only 36 experiments were designed, and an effective ANN model was trained. Using the Taguchi technique, additional combinations of experiments, not originally tested, were able to be predicted. The results obtained indicate that the best settings for the ANN parameters are learning function: GDM, training function: BFGS, umber of hidden layer: 5, number of neurons: 5 , type of ANN: MLP and transfer function: GDA. Additionally, contribution of the parameters in the regression model indicates that Nn and NL have the most percent contribution while LF has the least one. The results obtained demonstrate that the Taguchi method is an effective tool in identifying the optimal ANN parameters.

\section{References}

[1] Fateh, M. M. \& Khoshdel, V. (2015). Voltagebased adaptive impedance force control for a lowerlimb rehabilitation robot. Advanced Robotics, vol. 29, no. 15 , pp. 961-971.

[2] Khoshdel, V. \& Akbarzadeh Tootoonchi, (2015). A. Robust Impedance Control for Rehabilitation Robot. Modares Mechanical Engineering, vol. 15, no. 8, pp. 429-437.

[3] Jain, R. K., Datta, S. \& Majumder, S. (2013). Design and control of an IPMC artificial muscle finger for micro gripper using EMG signal. Mechatronics, vol. 23 , no. 3 , pp. 381 .

[4] Dellon, B. \& Matsuoka, Y. (2007). Prosthetics, exoskeletons, and rehabilitation (grand challenges of Robotics). IEEE Robot. Autom. Mag., vol. 14, no. 1, pp. 30-34.

[5] Wu, S., Waycaster, G. \& Shen, Xi. (2011). Electromyography-based control of active above-knee prostheses. Control Engineering Practice, vol 19, no. 8, pp. 875-882.

[6] Kiguchi, K., Kariya, S., Watanabe, K., Izumi, K. \& Fukuda, T. (2001). An exoskeletal robot for human elbow motion support-Sensor fusion, adapta tion, and control. IEEE Trans. Syst., Man, Cybern. B, Cybern., vol. 31, no. 3, pp. 353-361.

[7] Rosen, J., Brand, M., Fuchs, M. \& Arcan, M. (2001). A myosignal-based powered exoskeleton system. IEEE Trans. Syst., Man, Cybern. A, Syst., Humans, vol. 31, no. 3, pp. 210-222.
[8] Kiguchi, K., Rahman, M. H., Sasaki, M. \& Teramoto, K. (2008). Development of a 3DOF mobile exoskeleton robot for human upper limb motion assist," Robot. Autonom. Syst. vol. 56, no. 8, pp. 678691.

[9] Fleischer C. \& Hommel, G. (2008). A human exoskeleton interface utilizing electromyography. IEEE Trans. Robot. vol. 24, no. 4, pp. 872-882.

[10] Moritani, T. \& Muro, M. (1987). Motor unit activity and surface electromyogram power spectrum during increasing force of contraction. Eur $\mathrm{J}$ Appl Physiol Occup Physiol. vol. 56, no. 3, pp. 260-265.

[11] Hogan, N. \& Mann, R. W. (1980). Myoelectric signal processing, Optimal estimation applied to electromyography-Part1: Derivation of the optimal myoprocessors. IEEE Transactions on Biomedical Engineering, vol. 27, no. 7, pp. 396-410.

[12] Siegler S., Hillstrom H. J, Freedman, W., \& Moskowitz, G. (1985). Effect of myoelectric signal processing on the relationship between muscle force and processed EMG. Am. J. Phys Med, vol. 64, no. 3, pp. 130-149.

[13] Lin, T. Y, Ping, H. C., Hsu, T. H. \& Wang, L. C. (2011). A systematic approach to the optimization of artificial neural networks. In: IEEE 3rd International conference on communication software and networks (ICCSN), vol. 6, pp. 76-79.

[14] Inhira, E. \& Yokoi, H. (2007). An optimal design method for artificial neural networks by using the design of experiments. J Adv Comput Intell Inform, vol. 11, pp. 593-599.

[15] Kim, Y. S. \& Yum, B. J. (2004). Robust Design of Multilayer Feed Forward Neural Networks: An Experimental Approach. Appl. Artif. Intell., vol. 17, pp. 249-263.

[16] Packianather, M. S., Drake, P.R. \& Rowland, H. (2000). Optimizing the Parameters of Multilayered Feed Forward Neural Networks through Taguchi Design of Experiments. Qual. Reliab. Eng. Int., vol. 16, pp. 461-473.

[17] Yang, S. M. \& Lee, G. S. (1999). Neural Network Design by using Taguchi Method. J. Dyn. Syst. Meas. Contr, vol. 121, pp. 560-563.

[18] Khaw, J. F. C., Lim, B. S. \& Lim, L. E. N. (1995). Optimal Design of Neural Networks using the Taguchi Method. Neuralcomputing, vol. 7, pp. 225-245.

[19] Roy, R. K. (2001). Design of Experiments Using the Taguchi Approach: 16 Steps to Product and Process Improvement. Wiley, (2001).

[20] Lee, K. \& Kim, J. (2000). Controller gain tuning of a simultaneous multi-axis PID control system using the Taguchi method. Control Engineering Practice, vol. 8, no. 8, pp. 949-958. 